\title{
A Newly Developed Wearable Device for Continuous Measurement of Gait- Induced Accelerations in Daily Activities
}

Hiroshi Mitoma $^{1^{*}}$ and Mitsuru Yoneyama ${ }^{2}$

${ }^{1}$ Department of Medical Education, Tokyo Medical University, Japan

${ }^{2}$ MCHC R\&D Synergy Center Inc., Tokyo, Japan

*Corresponding author: Hiroshi Mitoma, M.D., Ph.D., Department of Medical Education, Tokyo Medical University, 6-7-1 Nishishinjuku, Shinjuku-ku, Tokyo 160-0023, Japan, Tel: +81-3-3342-6111-5632; Fax: +81-3-3342-5049; E-mail: mitoma@tokyo-med.ac.jp

Received date: May 31, 2014, Accepted date: June 18, 2014, Published date: June 26, 2014

Copyright: ( 2014 Mitoma H, et al. This is an open-access article distributed under the terms of the Creative Commons Attribution License, which permits unrestricted use, distribution, and reproduction in any medium, provided the original author and source are credited.

\begin{abstract}
For assessment of gait disorders, speed and stride are measured during 10m-walking test. However, changes in these two parameters are nonspecific since they are observed in various gait disorders. In addition, the10-m walking is short and reflects only one aspect of daily living and the results could be affected by emotional stress. To examine the step cycles and forces for step-in and kick off, which are directly controlled by a complex neural circuitry, during daily walking and over a long period of time, we developed a new wearing device, the portable gait rhythmogram (PGR), which monitors gait-induced accelerations for up to 70 hours. Quantitative analysis of the gait acceleration cycle and amplitude has allowed characterization of the bradykinematic features of gait disorders in patients with Parkinson's disease: 1) A decrease in amplitude of gait acceleration in the early stages of the disease, which is compensated by fast stepping. 2) Subjective motor fluctuation did not necessarily coincide with changes in gait parameters. The results suggest that the rhythms-force correlation is set by the basal ganglia, but can be modified by the cerebral cortex. Analysis of voluntary gait in daily life could enhance our understanding of the pathomechanisms of gait disorders.
\end{abstract}

Keywords: Gait disorder; Gait analysis; Wearable device; Parkinson's disease

\section{Background: Methodological Problems Related to Gait Analysis}

Gait disorders are cardinal neurological symptoms; locomotor disability negatively affects quality of daily life. The features of gait disorders can be clinically distinguished in various neurological diseases. For example, patients with Parkinson's disease (PD) walk with forward posture, small steps and slow cycle, frequently accompanied by freezing of gait and festination [1-3]. On the other hand, patients with vascular parkinsonism (VP) exhibit upright posture of the trunk and neck, stiff leg movements, short steps with frequent shuffling, slightly wide-based stance, and impaired postural reflexes that can lead to falls [4-9]. Patients with cerebellar ataxia (CA) walk with exaggerated swing of the trunk and wide-based stance; their gait cycles are slow and irregular $[1,2,10]$. Although gait disorder features have been defined clinically in each disease, the fundamental characteristics of various gait kinematic parameters have not yet been determined due to three methodological problems.

First, the majority of previous studies have documented a decrease in velocity and stride during walking. However, walking with short stride, wide stance, slow speed, and prolonged double support period is the final common expression of any dysfunction of the gait control system, not the characteristic change $[5,10]$. Thus, in addition to these nonspecific and secondary changes, the gait cycle or the floor reaction forces, parameters that are directly determined and controlled by neural circuits, should also be examined $[5,10]$
Second, short-distance walking is the only protocol that has been applied in previous studies [11]. One shortfall of short-distance walking is that the characteristic changes are easily compensated by emotional stress or augmented attention [12]. For example, freezing of gait in PD patients is unlikely to occur during a 10 -m walk test.

Third, tests based on short-distance walking can clarify only one aspect of gait movements in daily life. Gait movements are not automatic, but controlled voluntarily in daily life $[1,2]$. The step cycles and forces for step-in and kick-off are fine-tuned according to the context in which the gait movement is performed. Faster walking is associated with increased forces, with resultant long stride and fast steps, while careful walking along a narrow and uneven street is achieved with small forces and slow steps.

Accordingly, for proper assessment of the characteristic features of each gait disorder, it is important to measure the gait cycle and floor reaction forces over a long period of time during various gait movements in daily living. Long-term monitoring can trace changes in gait parameters in daily life, without emotional compensation, and hence provides important data on the mechanisms of deficits in voluntary control of gait movements.

\section{Methods: Gait Rhythmography for Monitoring Gait- Induced Accelerations}

A new device (portable gait rhythmogram, PGR) was developed for long-term monitoring of the gait cycle and/or force during walking in routine daily activity. The PGR is a trunk-mounted small device (size, $8 \times 6 \times 2 \mathrm{~cm}$, weight, $80 \mathrm{~g}$ ) (Figure 1) housing an acceleration sensor with automatic gait detection algorithm, in which gait induced- 
accelerations are extracted from limb and trunk movements using a mathematical algorithm; the "pattern matching method" [13-20].

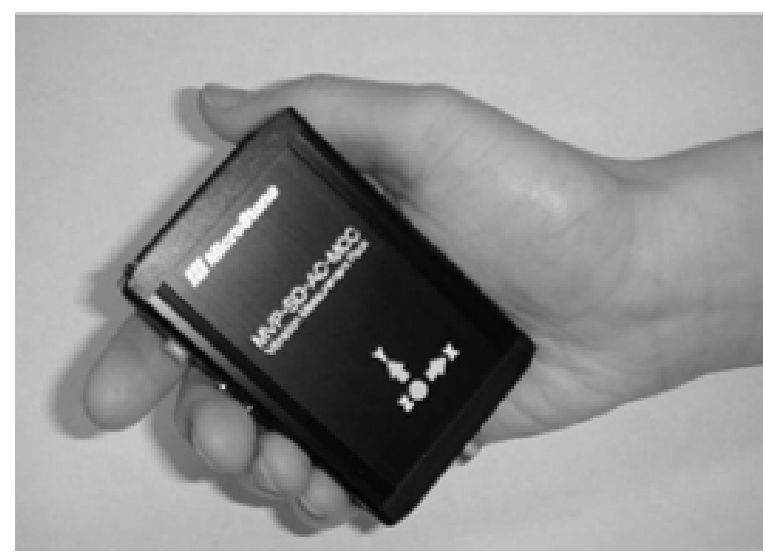

Figure 1: The portable gait rhythmogram device.

\section{Monitoring of motion-induced acceleration}

The PGR measures three dimensionally (ax, ay, az) 1) accelerations associated with limb and trunk movements, and 2) accelerations induced by step-in and kick-off during gait [13-16]. The PGR is attached to the waist of the patient, and records the above signals at a sampling rate of $10 \mathrm{msec}$. The data are automatically stored in a micro-SD card. After transfer of the recorded data to a PC, the absolute value of acceleration vectors $\left(a ; a^{2}=a x^{2}+a y^{2}+a z^{2}\right)$ is computed and graphically displayed on the monitor. A fully-charged PGR can achieve 70 hours of continuous recording.

\section{Identification of acceleration induced by gait motion}

The acceleration vectors associated with stepping can be distinguished from those associated with other limb and trunk movements or with unexpected artifacts, based on the mathematical method of "pattern matching", as reported in detail previously [13-16]. First, attention is focused on relatively strong signal region (e.g., a $>1$ $\mathrm{m} / \mathrm{s}^{2}$ ) in the acceleration time series, and a three-dimensional template wave (ax, ay, az) with a duration of about $0.5 \mathrm{sec}$ is arbitrarily chosen. Then, the cross-correlation $\mathrm{CC}(\mathrm{t})$ between this wave and another wave with a time shift $t$ chosen from the whole time series is computed by the following formula:

$$
\frac{1}{p}_{i=1}^{p}\left[a_{X}(i) a_{X}(i+t)+a_{Y}(i) a_{y}(i+t)+a_{z}(i) a_{z}(i+t)\right]
$$

$C C(t)=\overline{\left\{\frac{1}{p}_{i=1}^{p}\left[a_{X}(i)^{2}+a_{y}(i)^{2}+a_{Z}(i)^{2}\right]\right\}^{\frac{1}{2}}\left\{\frac{1}{p}_{i=1}^{p}\left[a_{X}(i+t)^{2}+a_{y}(i+t)^{2}+a_{Z}(i+t)^{2}\right]\right\}^{\frac{1}{2}}}$

Where $t$ is the time index and $p$ is the length of the template wave. If the acceleration change is caused by gait motion, the $\mathrm{CC}(\mathrm{t})$ peaks exhibit alternate changes in magnitude with time due to left/right body sway during walking.

\section{Estimation of gait cycle and acceleration}

The cycle and amplitude are measured from the gait-induced acceleration signals. Since gait accelerations correlate with floor reaction forces, the amplitude of gait accelerations is selected as an index of floor reaction forces. The duration of gait cycles and the amplitude of gait-related accelerations are averaged for $10 \mathrm{~min}$.

\section{Control experiment: Accuracy of PGR in detecting gait- induced signals}

In validation experiments using metronome-guided walking, 11 healthy subjects and $12 \mathrm{PD}$ patients were tested to evaluate the sensitivity of the PGR-algorithm. These studies showed detection of gait peaks with an accuracy of more than $94 \%$ in both groups of subjects $[14,15]$. Thus, our new gait analysis system can detect gaitinduced signals and define bradykinetic aspects even in daily walking of PD patients.

\section{Disturbance of Control Systems of Gait Cycle and Acceleration in PD}

Using PGR, we examined changes in gait cycle and amplitude of gait-induced acceleration during daily walking in $\mathrm{PD}$ patients. The results highlighted new aspects of disturbances of the gait control centers with advancement in PD [20]. PGR was recorded continuously over 24 hours in $64 \mathrm{PD}$ independent patients who engaged in activities of daily living, and 17 age-matched controls. The mean values of the gait cycle and acceleration were computed. The mean gait cycle throughout the day was significantly faster in PD patients with modified Hoehn and Yahr (mH\&Y) stage 1.5 than those with stages 2.5-3.0 and normal controls, but there was no significant difference in gait cycle between patients with stages 2.5-3.0 PD and normal controls. In contrast, the mean gait acceleration was significantly lower in PD patients than controls, and there were no significant differences among the disease stage groups.

These results suggest marked decrease in gait acceleration from the early stage of PD. Accumulating evidence suggests that the floor reaction force correlates with stride, and that shortened stride is due to a disproportionate force production $[14,15]$. Thus, at the early stage of $\mathrm{PD}(\mathrm{mH} \& \mathrm{Y}$ stage 1.5), the decrease in floor reaction force can result in shortened stride.

The shortened stride can be compensated by fast stepping. In a series of studies, Morris et al. [21-23] proposed that a decrease in stride length was an important change in parkinsonian walking and that PD patients walk with a faster gait cycle to maintain overall speed. Based on this concept of compensatory changes, the results reported in our previous study [20] could be explained as follows: 1) while the control center for the step cycle is preserved (at the early stage, $\mathrm{mH} \& \mathrm{Y}$ stage 1.5), efficient compensation occurs, that is, shortness of stride is compensated by cycle acceleration. 2) moderate disturbance of the center responsible for the control of the step cycle (i.e., as seen in patients with $\mathrm{mH} \& \mathrm{Y}$ stages 2.0-3.0), results in difficulties in speeding up the gait cycle due to lack of efficient compensation, and thus the gait cycle in these patients would be similar to that of the normal subjects. 3) Severe disturbance of the center involved in the control of the step cycle is associated with failure of compensation, leading to bradykinesia. Consistent with this hypothesis, we reported previously slowing of the gait cycle during the wearing-off period in patients with prominent bradykinesia $[13,17]$. The results also suggest that the gait cycle and acceleration of gait movements are controlled independently and that disturbances in these movements exhibit different clinical courses in PD. 


\section{Subjective Motor Fluctuation in PD Does Not Necessarily Correlate with Gait Fluctuation}

The clinicians cannot always be aware of what happens to patients outside the hospital. Based on the capability of the PGR for long-term monitoring, we monitored changes in gait abilities in PD patients. The continuous recording provided new findings on motor fluctuation in PD $[13,19]$.

Dopamine-replacement therapy at the early stage of PD decreases motor and non-motor complications. In the advanced stage, however, motor fluctuation is a frequent and disabling problem. Up to $50 \%$ of patients exhibit motor fluctuation and resistance to medication after the first five years of treatment [12,24,25]. Motor fluctuation is estimated by history taking or patients' diaries, though such information is subjective and not quantitative [26,27]. Like many other medical conditions, some patients either under- or over-rate their symptoms and signs, making it difficult to assess the extent of actual motor fluctuation.

To provide a better assessment of gait while the patient is at home and for quantitative evaluation of gait fluctuation, we recorded gait cycle and acceleration continuously over the 24-hr period in 54 patients with PD and 17 controls [19]. The patients were also asked to estimate motor fluctuation every hour with diaries. In 44 of 54 patients, changes in the cycle were associated with simultaneous changes in the acceleration. We examined the subjective fluctuation in these 44 patients who showed synchronization between cycles and accelerations (matched patients). In this group of 44 matched patients, 23 patients did not report any fluctuations in their diaries. However, the PGR detected gait disorders in 19 of these 23 patients (82.7\%) who reported no complaints in their diaries. During off time, they walked with marked short- or bradykinetic-stepping (Figure 2A). On the other hand, 21 of the 44 matched patients perceived motor fluctuation. However, no matching changes were observed in either the cycle or acceleration in $11(52.4 \%)$ of these 21 patients (Figure 2B). Consequently, no synchronization was noted in $30(68.2 \%)$ of the 44 matched patients, between the times of subjectively assessed motor fluctuation and those of quantitative analysis of gait disorder. This discrepancy highlights the clinical importance of continuous recording of the cycle and acceleration in the assessment of motor fluctuation.

What is the reason for the lack of synchronization? One reason for the discrepancy could be that the concerns or priorities varied from one patient to another. In some patients, the target of attention might be other types of movement disorders. For example, some patients might feel good when they can easily manipulate their hands. Other patients might feel subjectively good upon improvement of hypokinesia (difficulty in initiation of movements). In such patients, they would estimate to be on time when they easily initiated their movements without hesitation. Moreover, other patients might weigh mood disorders, such as apathy, rather than movement disorders. Thus, the present finding of desynchronization between subjective off and gait off could represent the inherent features of PD itself. Another alternative explanation is that some PD patients did not notice motor fluctuation due to deficits in attention to motor deficits [27]. In such a condition, fluctuation in gait parameters would have occurred even though the patients felt good throughout the day.

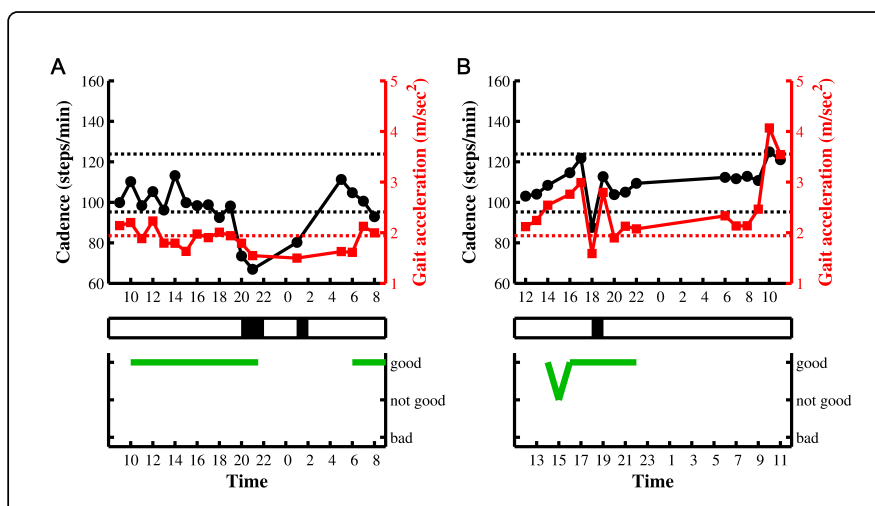

Figure 2: Comparison between gait fluctuation (top) and subjective fluctuation (bottom). Top: serial changes in gait cadence (steps/ $\mathrm{min}$ ) and acceleration during daily activities. Left ordinate: cadence (black continuous line and symbols), right ordinate: acceleration (red continuous line and symbols), abscissa: time, black dotted line: mean cadence $\pm 1 S D$ of normal subjects, red dotted line: mean acceleration - 2SD of normal subjects. The determined gait off is indicated by the black rectangle below the top Figure. In (a), the patient subjectively noticed no fluctuation, whereas the gait disorders occurred at 20-22. In (b), the patient subjectively noticed off at 15 , whereas the gait worsened at 18 . No synchronization was noted in $30(68.2 \%)$ of the tested 44 matched patients, between the times of subjectively assessed motor fluctuation and those of quantitative analysis of gait disorder.

\section{Physiological Considerations: How Are the Gait Cycle and Floor Reaction Forces Determined in Neural Circuitry?}

Continuous recordings of walking movements during daily activities also clarified how gait parameters are voluntarily controlled. We proposed the technique of gait acceleration-cycle plot, to examine how the gait is voluntarily controlled $[15,17,18]$.

The duration of gait cycles and the amplitude of gait-related accelerations were averaged for $10 \mathrm{~min}$ recording and plotted (Figure 3).

Figure 3 shows the gait acceleration-cycle plot of each PD patient with the corresponding regression line (thick line). The averaged logarithmic values showed normal distribution and the slope of the regression line was calculated using principal component analysis. The thin regression line was computed from the summed average of 17 controls.

The graphs shown in Figure 3 indicate that changes in the gait cycle duration correlated with those in the gait acceleration amplitude. That is, walking with fast gait acceleration, i.e., large floor reaction forces, was associated with faster gait cycle. Interestingly, all relations between the gait acceleration amplitude and duration of gait cycle during the test day were represented in one or two regression lines. 

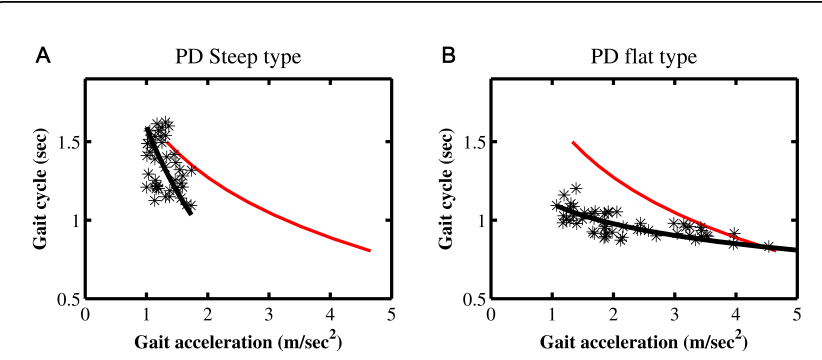

Figure 3: Examples of gait acceleration-cycle relationship in a patient with Parkinson's disease (PD) $(\mathrm{a}, \mathrm{b})$ based on 24-hr gait recording. The corresponding regression line is shown by thick line. In (a) and (b), the regression line, obtained from the summed average of 17 normal controls, is also shown (red thin line). The regression line of the PD patient is steep in (a), whereas it is flat in (b)

\section{Gait acceleration-cycle plot patterns in PD patients}

The characteristic change in the shape of this curve is the narrowness of the range of force and/or rhythm [17,18]. In PD patients who walked with a narrow range of change in gait acceleration (i.e., floor reaction forces), the slope of the regression curve was steep (steep type) (Figure 3A). This type reflects short-step walking pattern, in which no effective floor reaction force is achieved despite the fast step. In contrast, PD patients who walked with a narrow range of change in the gait cycle, the regression curve was flat (flat type) (Figure 3B). Under this condition, the patient always walks with the preferred cycle, i.e., monotone walking. Other patients walked with a narrow range of both acceleration and cycle, with small scatter of gait acceleration and cycle data (lump type).

For quantitative assessment of these changes, we used the index of $\%$ alteration range [18]. In the gait acceleration-step cycle plot, which was obtained from one trial in each patient, the mean and standard deviation for gait acceleration and cycle were calculated. Here, the SD was determined for an index of the alteration range. The interindividual mean of SD was calculated from data of 17 controls. Thus, the ratio of the SD for each patient to the inter-individual mean of SD for the normal controls was defined as $\%$ alteration range. In analysis of the \%alteration range, the cutoff level was set at $75 \%$ and examined 40 patients with PD. The regression curves of patients with \%alteration range $>75 \%$ were similar to those of the controls $(n=16,40 \%)$. Twelve (30\%) patients showed gait acceleration-cycle plots of patients with \%alteration range of gait acceleration of $<75 \%$ (steep type), whereas 8 (20\%) patients showed gait acceleration-cycle plots of patients with $\%$ alteration range of gait cycle of $<75 \%$ (flat type). The lump pattern was noted in 4 patients (10\%).

Flowers [28] postulated that PD patients can only execute movements of different amplitudes at a single, slow, velocity and cannot increase their movement velocity. Consistent with this notion, the peak EMG activity that can be generated in a muscle burst is limited in PD patients [29]. Thus, the present finding seems also to suggest that PD patients cannot vary the force and rhythm in their daily routine walking, and that they consequently walk with a narrow range of floor reaction forces and step cycles.

\section{Gait acceleration-cycle plot patterns in normal subjects}

Various patterns of waling may be at work under daily walking of normal controls [16]. Figure 4 shows plots from a representative normal subject, in which two regression lines were needed for better fitting, suggesting the existence of two patterns of walking. The steep regression curve shows that a subject walked with small accelerations for a particular cycle, suggesting that he walked quietly, for example in the room. In contrast, the flat regression curve shows that the subject walked with large accelerations for a particular cycle. Under this condition, he would walk hard, for example, outside the house. The two patterns were also observed in 8 out of 17 normal subjects.

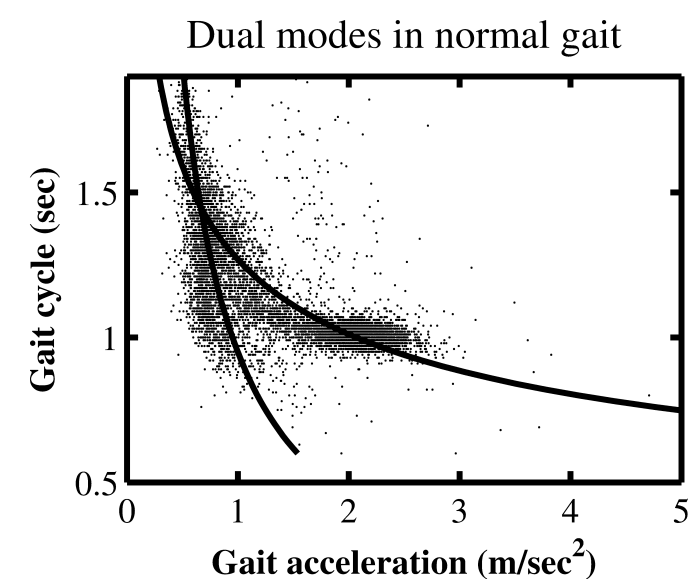

Figure 4: Examples of gait acceleration-cycle relationship in a normal subject based on 24-hr gait recording. Here, the duration of gait cycles and the amplitude of gait-related accelerations were plotted for all steps. Two regression lines are needed for better fitting.

\section{How is the gait strategy selected by neural circuitry?}

These two sets of results obtained from patients with PD and normal subjects suggest the following scheme. 1) The basal ganglia seem to set the relation between the cycle and the force. Especially, the basal ganglia seem to play a critical role in the formation of the wide dynamic range of these parameters. The rhythm generators in the brainstem or the spinal cord are under the control of these settings. 2) The cerebral cortex seems to modify the relation between the cycle and the force, formulated in the basal ganglia, depending on the context in which walking movements are performed. This idea is in good agreement with the recent findings revealed by new imaging techniques [30-32]. These studies showed that white matter lesions disrupted balance/locomotor circuits and thereby caused the highlevel gait disorders characterized by deficits in strength, tone, sensation or coordination. Our working hypothesis should be further tested by continuous recordings of various types of gait disorders in daily activities.

\section{Conclusion}

Our newly developed PGR device has the following properties: (1) the capacity of long-term monitoring (more than two days), and (2) the exact measurements of gait-induced accelerations from other movements, when compared to other portable devices [33]. The 
advantages of the PGR allowed us to capture changes in gait cycles and accelerations in daily walking of patients with PD. These changes reflected the clinical course of parkinsonian gait disorder and motor fluctuation. Furthermore, the analysis using gait acceleration-cycle plots provided clues about neural control of gait rhythm and forces. Further analysis of voluntarily controlled daily walking would should enhance our understanding of the pathomechanisms of various gait disorders and the development of better therapies.

\section{References}

1. Dietz V (1992) Human neuronal control of automatic functional movements: interaction between central programs and afferent input. Physiol Rev 72: 33-69.

2. Dietz V (1997) Neurophysiology of gait disorders: present and future applications. Electroencephalogr Clin Neurophysiol 103: 333-355.

3. Schaafsma JD, Balash Y, Gurevich T, Bartels AL, Hausdorff JM, et al. (2003) Characterization of freezing of gait subtypes and the response of each to levodopa in Parkinson's disease. Eur J Neurol 10: 391-398.

4. Critchley M (1929) Arteriosclerotic parkinsonism. Brain 52: 23-83.

5. Nutt JG, Marsden CD, Thompson PD (1993) Human walking and higher-level gait disorders, particularly in the elderly. Neurology 43: 268-279.

6. Thompson PD, Marsden CD (1987) Gait disorder of subcortical arteriosclerotic encephalopathy: Binswanger's disease. Mov Disord 2: 1-8.

7. Thompson PD (2001) Gait disorders accompanying diseases of the frontal lobes. Adv Neurol 87: 235-241.

8. Nutt JG (1988) Parkinson's disease and movement disorders: Gait disorders. Urban \& Schwarzenberg, Baltimore.

9. Fitz Gerald PM, Jankovic J (1989) Lower body parkinsonism: evidence for vascular etiology. Mov Disord 4: 249-260.

10. Mitoma H, Hayashi R, Yanagisawa N, Tsukagoshi H (2000) Characteristics of parkinsonian and ataxic gaits: a study using surface electromyograms, angular displacements and floor reaction forces. J Neurol Sci 174: 22-39.

11. Knutsson E (1972) An analysis of Parkinsonian gait. Brain 95: 475-486.

12. Yanagisawa N (2006) Natural history of Parkinson's disease: From dopamine to multiple system involvement. Parkinsonism Relat Disord 12: S40-46.

13. Mitoma H, Yoneyama M, Orimo S (2010) 24-hour recording of parkinsonian gait using a portable gait rhythmogram. Intern Med 49: 2401-2408.

14. Yoneyama M, Kurihara Y, Watanabe K, Mitoma H (2014) Accelerometry-based gait analysis and its application to Parkinson's disease assessment--part 1: detection of stride event. IEEE Trans Neural Syst Rehabil Eng 22: 613-622.

15. Yoneyama M, Kurihara Y, Watanabe K, Mitoma H (2013) Accelerometry-based gait analysis and its application to Parkinson's disease assessment- part 2: a new measure for quantifying walking behavior. IEEE Trans Neural Syst Rehabil Eng 21: 999-1005.

16. Yoneyama M, Mitoma H, Okuma Y (2013) Accelerometry-based longterm monitoring of movement disorders: from diurnal gait behavior to nocturnal bed mobility. J Mech Med Biol 13: 1350041.
17. Utsumi H, Terashi H, Ishimura Y, Takazawa T, Hayashi A, et al. (2012) Quantitative assessment of gait bradykinesia in Parkinson's disease using a portable gait rhythmogram. Acta Med Okayama 66: 31-40.

18. Terashi H, Utsumi H, Ishimura Y, Takazawa T, Okuma Y, et al. (2012) Deficits in scaling of gait force and cycle in parkinsonian gait identified by long-term monitoring of acceleration with the portable gait rhythmogram. ISRN Neurol 2012: 306816.

19. Utsumi H, Terashi H, Ishimura Y, Takazawa T, Okuma Y, et al. (2012) How far do the complaints of patients with Parkinson's disease reflect motor fluctuation? Quantitative analysis using a portable gait rhythmogram. ISRN Neurol 2012: 372030.

20. Terashi H, Utsumi H, Ishimura Y, Mitoma H (2013) Independent regulation of the cycle and acceleration in parkinsonian gait analyzed by a long-term daily monitoring system. Eur Neurol 69: 134-141.

21. Morris ME, Iansek R, Matyas TA, Summers JJ (1994) The pathogenesis of gait hypokinesia in Parkinson's disease. Brain 117: 1169-1181.

22. Morris ME, Iansek R, Matyas TA, Summers JJ (1994) Ability to modulate walking cadence remains intact in Parkinson's disease. J Neurol Neurosurg Psychiatry 57: 1532-1534.

23. Morris ME, Iansek R, Matyas TA, Summers JJ (1996) Stride length regulation in Parkinson's disease. Normalization strategies and underlying mechanisms. Brain 119:551-568.

24. Marsden CD, Parkes JD, Quinn N (1982) Fluctuations of disability in Parkinson's disease: clinical aspects: Movement disorders. Butterworth Scientific, London.

25. Giladi N (2008) Medical treatment of freezing of gait. Mov Disord 23 Suppl 2: S482-488.

26. Stacy M, Bowron A, Guttman M, Hauser R, Hughes K, et al. (2005) Identification of motor and nonmotor wearing-off in Parkinson's disease: comparison of a patient questionnaire versus a clinician assessment. Mov Disord 20: 726-733.

27. Stacy M, Hauser R (2007) Development of a Patient Questionnaire to facilitate recognition of motor and non-motor wearing-off in Parkinson's disease. J Neural Transm 114: 211-217.

28. Flowers KA (1976) Visual "closed-loop" and "open-loop" characteristics of voluntary movement in patients with Parkinsonism and intention tremor. Brain 99: 269-310.

29. Hallett M, Khoshbin S (1980) A physiological mechanism of bradykinesia. Brain 103: 301-314.

30. Tessitore A, Amboni M, Cirillo G, Corbo D, Picillo M, et al. (2012) Regional gray matter atrophy in patients with Parkinson disease and freezing of gait. AJNR Am J Neuroradiol 33: 1804-1809.

31. Nutt JG (2013) Higher-level gait disorders: an open frontier. Mov Disord 28: $1560-1565$.

32. Bolandzadeh N, Liu-Ambrose T, Aizenstein H, Harris T, Launer L, et al. (2014) Pathways linking regional hyperintensities in the brain and slower gait. Neuroimage .

33. Maetzler W, Domingos J, Srulijes K, Ferreira JJ, Bloem BR (2013) Quantitative wearable sensors for objective assessment of Parkinson's disease. Mov Disord 28: 1628-1637. 